\title{
CONVERGENCE AND QUASI-OPTIMAL COMPLEXITY OF A SIMPLE ADAPTIVE FINITE ELEMENT METHOD
}

\author{
Roland Becker ${ }^{1}$ AND SHIPENG MAO ${ }^{2}$
}

\begin{abstract}
We prove convergence and quasi-optimal complexity of an adaptive finite element algorithm on triangular meshes with standard mesh refinement. Our algorithm is based on an adaptive marking strategy. In each iteration, a simple edge estimator is compared to an oscillation term and the marking of cells for refinement is done according to the dominant contribution only. In addition, we introduce an adaptive stopping criterion for iterative solution which compares an estimator for the iteration error with the estimator for the discretization error.
\end{abstract}

Mathematics Subject Classification. 65N12, 65N15, 65N30, 65N50.

Received July 27, 2008. Revised April 17, 2009.

Published online August 21, 2009.

\section{INTRODUCTION}

The analysis of adaptive finite element methods has made important progress in recent years. Based on classical residual-based a posteriori error estimators $[1,16,20]$ it has been shown by Dörfler et al. $[14,15,17]$ that an adaptive mesh refinement algorithm converges towards the solution of the Poisson equation with homogeneous Dirichlet boundary conditions and given square-integrable forces $f$ in a two-dimensional bounded domain $\Omega$ with piecewise linear boundary $\partial \Omega$ :

$$
-\Delta u=f \quad \text { in } \quad \Omega, \quad u=0 \quad \text { on } \quad \partial \Omega
$$

In [17] the importance of controlling oscillations in data not captured on a given finite element mesh is pointed out and a special local refinement algorithm ('newest-vertex-bisection') is used in order to control a term measuring the data oscillations.

An important further result is the estimation of the dimension of the adaptively constructed discrete spaces, first achieved for wavelet discretizations by Cohen et al. [12]. This result is extended to a modified version of the algorithm of [17], including an additional coarsening step, by Binev et al. in [6]. A further significant improvement has been achieved by Stevenson [19] who shows that the additional coarsening step is not necessary in order to prove optimal complexity.

Keywords and phrases. Adaptive finite elements, a posteriori error analysis, convergence of adaptive algorithms, complexity estimates.

1 Laboratoire de Mathématiques Appliquées and INRIA Bordeaux Sud-Ouest Concha, Université de Pau, 64013 Pau Cedex,

France. roland.becker@univ-pau.fr; beckerroland@me.com

2 Institute of Computational Mathematics and INRIA Bordeaux Sud-Ouest Concha, Chinese Academy of Sciences (CAS),

Beijing, 100190, P. R. China. maosp@lsec.cc.ac.cn 
The importance of the last-mentioned results lays in the fact that they show optimal complexity of certain adaptive algorithms: if the solution of (1.1) can be approximated by a given discretization method on a given family of meshes at a certain rate (quotient of accuracy to number of unknowns), the iteratively constructed sequence of meshes will realize this rate up to a constant factor.

The results obtained in the cited works rely on a special treatment of the before-mentioned data oscillation term and the use of the newest-vertex-bisection algorithm. To overcome the need of this special refinement algorithm, which is cumbersome for practical purposes, is a difficult task, since the classical local lower bound, which is at the heart of convergence proofs, has to be avoided. A first result in this direction has been recently proven in [18], which does however not provide any information on speed of convergence nor complexity of the algorithm. More recently, in [10] the authors provide a convergence and complexity result for an adaptive algorithm avoiding the inner node property. The idea is to replace the local lower bound by a decrease estimate of the error estimator.

Our algorithm is based on an adaptive marking strategy comparing an oscillation term with a simple estimator, following [5] where this idea has been introduced in the context of the new vertex bisection and [2] for mixed finite elements. In the present work, we avoid the inner node property, which makes it possible to use other local refinement algorithms, supposed that they satisfy a complexity estimate stated below. With respect to the cited articles, we simplify the a posteriori error estimator by suppressing the volume residual. This is natural, since it is known for a long time that the volume term leads to overestimation in the case of lowest-order conforming finite elements $[8,9]$. The resulting algorithm is attractive in practice, since the obtained refinement will in general be dominated by the edge residuals.

In addition, we introduce a new adaptive stopping criterion for the iterative solution of the discrete system in each step of the mesh refinement algorithm. The idea of this stopping criterion is to compare the iteration error $\varepsilon_{\text {it }}$ with the estimator for the discretization error. This strategy potentially saves important computing time, since criteria with a fixed small constant, say $\varepsilon_{i t} \leq 10^{-8}$, often used in practice, require close to complete solution in each step. We analyze the resulting algorithm with incomplete iterative solution and prove convergence and optimal complexity.

Throughout this paper we work with families of shape regular triangular meshes in the sense of [11]. In order to deviate as less as possible from standard notation, we denote by $h$ an element of a family of admissible meshes $\mathcal{H}$, and by $u_{h}$ the corresponding finite element solution. The set of cells of mesh $h$ is denoted by $\mathcal{K}_{h}$, and the set of interior edges by $\mathcal{E}_{h}$. In addition, the set of nodes is denoted as $\mathcal{N}_{h}$. As compared to standard notation in finite element literature, $h$ denotes a mesh in a family of meshes $\mathcal{H}$ and not a global maximal cell width.

The paper is organized as follows: In Section 2 we define the adaptive algorithm. In Section 3 we prove some lemmata concerning lower/upper local/global bounds which are used later. Although the techniques used here are well established in the literature, we give complete proofs since we need to elaborate the precise dependence on iterative solution for the purpose of the subsequent analysis. In Section 4 we prove geometrical convergence of the error of the adaptive algorithm, under natural assumptions. In Section 5 we prove an asymptotic estimate for the complexity of the sequence of generated meshes. Possible concrete forms of the stopping criterion are discussed in Section 6. In Section 7 we present some numerical experiments and some conclusions are drawn in Section 8.

Throughout the paper we use the following notation. We use the standard Sobolev space $H_{0}^{1}(\Omega)$ and write for $u \in H_{0}^{1}(\Omega)$ and $\omega \subset \Omega|u|_{1, \omega}:=\left(\int_{\omega}|\nabla u|^{2} \mathrm{~d} x\right)^{1 / 2}$ and $|u|_{1}=|u|_{1, \Omega}$. The $L^{2}(\omega)$-scalar product is denoted by $\langle\cdot, \cdot\rangle_{\omega}$ with corresponding norm $\|\cdot\|_{\omega}$, omitting the subscript in case $\omega=\Omega$.

Furthermore, $P^{k}$ denotes the polynomials of maximal degree $k \geq 0$.

\section{Definition of the ADAPtive ALGORIthm}

We define the family of admissible meshes $\mathcal{H}$ in the following recursive way. Starting from an initial mesh $h_{0}$, we suppose that we have a local mesh refinement algorithm $\mathcal{R}_{\text {loc }}(h, \mathcal{F})$ which refines for a given mesh $h \in \mathcal{H}$ 
all marked edges $\mathcal{F} \subset \mathcal{E}_{h}$. The resulting mesh is supposed to be conforming and the local mesh refinement algorithm is supposed to satisfy the following assumption. Let us denote by $N_{h}=\# \mathcal{K}_{h}$ the number of cells of the mesh $h$. Then we require that:

Assumption 2.1. Let $h_{k}, k=0, \ldots, n$ be a sequence of locally refined triangulations created by the local mesh refinement algorithm, starting from the initial mesh $h_{0}$. Let $\mathcal{F}_{k} \subset \mathcal{E}_{h_{k}}, k=0, \ldots, n-1$ be the collection of all marked edges in step $k$. Then $h_{n}$ is uniformly shape regular and its shape regularity only depends on that of $h_{0}$ and for all $E \in \mathcal{F}_{k}$ all neighboring triangles of $E$ are at least bisected once. Furthermore, there exists a mesh-independent constant $C_{0}$ such that

$$
N_{h_{n}} \leq N_{h_{0}}+C_{0} \sum_{k=0}^{n-1} \# \mathcal{F}_{k}
$$

Assumption 2.1 and especially the complexity estimate (2.1) are known to be true for the newest vertex bisection algorithm, see Theorem 2.4 of [6] (where the set of marked cells instead of the set of marked edges is used).

Let $h \in \mathcal{H}$. We use the Courant finite element space

$$
V_{h}:=\left\{v_{h} \in H_{0}^{1}(\Omega):\left.v_{h}\right|_{K} \in P^{1} \text { for all } K \in \mathcal{K}_{h}\right\}
$$

The Ritz projection $u_{h} \in V_{h}$ is defined by

$$
\left\langle\nabla u_{h}, \nabla v_{h}\right\rangle=\left\langle f, v_{h}\right\rangle \quad \forall v_{h} \in V_{h}
$$

In order to approximately solve the discrete system in (2.2), we use a multigrid algorithm, which is based on the sequence of nested spaces $V_{1} \subset \ldots \subset V_{l} \subset \ldots \subset V_{L}$ with $V_{L}=V_{h}$ resulting from the hierarchical mesh refinement. The multigrid algorithm employs mesh transfer operators and a smoothing operator only acting on the locally refined meshes, see $[7,21]$ for detailed definitions and proofs of the mesh-independent convergence of the resulting algorithms.

We denote by $u_{h}^{m}$ the discrete function obtained from $m$ iterations of the multigrid algorithm starting with the solution from the previous step of the mesh-refinement algorithm. In order to estimate the iteration error, we use an a posteriori error estimator for the definition of the stopping criterion of the multigrid iteration. Let $\zeta_{h}\left(u_{h}^{m}\right)$ be an estimator satisfying the upper bound

$$
\left|u_{h}-u_{h}^{m}\right|_{1}^{2} \leq C_{m g} \zeta_{h}^{2}\left(u_{h}^{m}\right)
$$

Several estimators satisfying (2.3) are possible, see Section 6 for a detailed discussion. Our results on convergence and complexity of the adaptive algorithm do not dependent on the concrete form of the estimator but only (2.3).

Let $h \in \mathcal{H}$ and let $\omega_{z}$ be the set of cells joining a node $z \in \mathcal{N}_{h}$ and denote by $\pi_{\omega}$ the mean-value operator $\left(\pi_{\omega}(f):=\int_{\omega} f \mathrm{~d} x /|\omega|\right)$. We define for given $z \in \mathcal{N}_{h}$ and $\mathcal{P} \subset \mathcal{N}_{h}$ an oscillation term

$$
\operatorname{osc}_{z}:=\left|\omega_{z}\right|^{1 / 2}\left\|f-\pi_{\omega_{z}} f\right\|_{\omega_{z}}, \quad \operatorname{osc}_{h}(\mathcal{P}):=\left(\sum_{z \in \mathcal{P}} \operatorname{osc}_{z}^{2}\right)^{1 / 2}
$$

Next we define for $E \in \mathcal{E}_{h}$ and any given subset $\mathcal{F} \subseteq \mathcal{E}_{h}$ the standard edge residuals for a given function $v_{h} \in V_{h}$ :

$$
J_{E}\left(v_{h}\right):=|E|^{1 / 2}\left\|\left[\frac{\partial v_{h}}{\partial n}\right]\right\|_{E}, \quad J_{h}\left(v_{h}, \mathcal{F}\right):=\left(\sum_{E \in \mathcal{F}} J_{E}^{2}\left(v_{h}\right)\right)^{1 / 2},
$$

where $[\cdot]_{E}$ denotes as usual the jump of a cell-wise polynomial function. We set for brevity $\operatorname{osc}_{h}:=\operatorname{osc}_{h}\left(\mathcal{N}_{h}\right)$ and $J_{h}\left(v_{h}\right):=J_{h}\left(v_{h}, \mathcal{E}_{h}\right)$. 
The purpose of this article is to analyze the following adaptive finite element algorithm:

Algorithm $1 \mathcal{A F E \mathcal { M }}$

(0) Choose parameters $0<\theta, \sigma<1, \gamma>0, \alpha>0$ and an initial mesh $h_{0}$, and set $k=0$.

(1) Do $m$ iterations of the iterative solution algorithm applied to the discrete system (2.2) with $h$ replaced by $h_{k}$ to obtain the finite element solution $u_{k}^{m} ; m$ is determined by the condition to be the smallest integer verifying:

$$
\zeta_{h_{k}}^{2}\left(u_{k}^{m}\right) \leq \alpha J_{h_{k}}^{2}\left(u_{k}^{m_{k}}\right)
$$

(2) Compute the a posteriori error estimator $J_{h_{k}}$ and oscillation term $\operatorname{osc}_{h_{k}}$.

(3) $\quad-$ If $\operatorname{osc}_{h_{k}}^{2} \leq \gamma J_{h_{k}}^{2}\left(u_{h_{k}}^{m}\right)$ then mark a set $\mathcal{F} \subset \mathcal{E}_{h_{k}}$ with minimal cardinality such that

$$
J_{h_{k}}^{2}(\mathcal{F}) \geq \theta J_{h_{k}}^{2}
$$

- else find a set $\mathcal{P} \subset \mathcal{N}_{h_{k}}$ with minimal cardinality such that

$$
\operatorname{osc}_{h_{k}}^{2}(\mathcal{P}) \geq \sigma \operatorname{osc}_{h_{k}}^{2}
$$

and define $\mathcal{F}$ to be the set of edges containing at least one node in $\mathcal{P}$.

(4) Adapt the mesh: $h_{k+1}:=\mathcal{R}_{\text {loc }}\left(h_{k}, \mathcal{F}\right)$.

(5) Set $k:=k+1$ and go to step (1).

Remark 2.2. The refinement is only governed by the oscillation term, if it is big compared to the estimator, following the idea of [5]. Therefore, in most practical cases, the edge residuals alone dominate the error estimation, such as suggested in the work of Carstensen and Verfürth [9].

Remark 2.3. The choice of parameters can be guided by our theoretical results. The stopping criterion $\alpha$ has to be small enough relative to $\theta$ in order to yield convergence; the other parameters $\theta, \sigma$, and $\gamma$ are arbitrary. The fact that $\gamma$ is arbitrary for our convergence result indicates that the edge residuals play the dominant role in the overall refinement.

In order to achieve optimal complexity, in addition to the condition on $\alpha$, the marking parameter $\theta$ has to be small enough and $\gamma$ has to satisfy a condition, whereas $\sigma$ is free.

Such a condition on $\theta$ is known from other complexity estimates [19]. In [6], $\theta=1$ is possible, but an additional coarsening step is used.

\section{Some technical Lemmata}

For the purpose of the later convergence and complexity proofs, we collect here some lemmata concerning upper and lower bounds of the estimators. Although the techniques used here are well established in the literature, see $[8-10,17,19]$, we give complete proofs since the subsequent analysis requires to elaborate the precise dependence on iterative solution.

Lemma 3.1 (upper bounds). Let $h \in \mathcal{H}$. There exists a constant $C_{1}>0$ depending only on the minimum angle of $h_{0}$ such that for $u_{h} \in V_{h}$ the solution of (2.2) and arbitrary $w_{h} \in V_{h}$

$$
\left|u-w_{h}\right|_{1}^{2} \leq C_{1}\left(J_{h}^{2}\left(w_{h}\right)+\operatorname{osc}_{h}^{2}\right)+2\left|u_{h}-w_{h}\right|_{1}^{2} .
$$

Suppose in addition that $H \in \mathcal{H}$ and $\mathcal{F} \subset \mathcal{E}_{H}$ are such that $h=\mathcal{R}_{\text {loc }}(H, \mathcal{F})$. Letting $\mathcal{P} \subset \mathcal{N}_{H}$ the set of nodes included in $\mathcal{F}$ and $u_{H} \in V_{H}$ the discrete solution, we have

$$
\left|u_{h}-w_{H}\right|_{1}^{2} \leq C_{1}\left(J_{H}^{2}\left(w_{H}, \mathcal{F}\right)+\operatorname{osc}_{H}^{2}(\mathcal{P})+\left|u_{H}-w_{H}\right|_{1}^{2}\right) \quad \forall w_{H} \in V_{H},
$$

and

$$
\# \mathcal{F} \leq C_{3}\left(N_{h}-N_{H}\right)
$$


Proof. The global upper bound (3.1) with $w_{h}=u_{h}$ has been proven by Carstensen in [8] introducing a weighted Clément-type quasi-interpolation operator, denoted by $C_{h}$. The generalization to $w_{h} \neq u_{h}$ follows from the triangle inequality.

For the proof of the localized upper bound (3.2) we make use of the operator $C_{h}$ which allows for the local interpolation estimates, known from the Clément-operator. First, there exists a mesh-independent constant $C_{\text {int }}$ such that

$$
\max \left(\left|v-C_{h} v\right|_{K},|K|^{1 / 4}\left|v-C_{h} v\right|_{\partial K}\right) \leq C_{\text {int }}|K|^{1 / 2}|v|_{1, \omega_{K}},
$$

where $\omega_{K}$ is the union of the neighboring elements. In addition, $C_{h}$ is constructed in such a way that, with another mesh independent constant $C_{\text {orth }}$,

$$
\left\langle f, v-C_{h} v\right\rangle \leq C \sum_{z \in \mathcal{N}_{H}}\left|\omega_{z}\right|\left|f-\pi_{\omega_{z}} f\right|_{\omega_{z}}|v|_{1, \omega_{z}} \leq C_{\text {orth }} \operatorname{osc}_{H}|v|_{1} .
$$

Let now $v_{H} \in V_{H}$ denote the quasi-interpolant of $v_{h}=u_{h}-w_{H} \in V_{h}$. Then the support of $v_{h}-v_{H}$ is located to the locally refined region augmented by one layer of elements. Therefore we obtain the bound (3.3) with $\mathcal{F}$ to be defined as the set of edges belonging to the support of $v_{h}-v_{H}$ :

$$
\begin{aligned}
\left|u_{h}-w_{H}\right|_{1}^{2} & =\left\langle\nabla\left(u_{h}-w_{H}\right), \nabla\left(v_{h}-v_{H}\right)\right\rangle+\left\langle\nabla\left(u_{H}-w_{H}\right), \nabla v_{H}\right\rangle \\
& \leq\left\langle f, v_{h}-v_{H}\right\rangle-\left\langle\nabla w_{H}, \nabla\left(v_{h}-v_{H}\right)\right\rangle+\left|u_{H}-w_{H}\right|_{1}\left|v_{H}\right|_{1} \\
& \leq\left(C_{\text {orth }} \operatorname{Osc}_{H}(\mathcal{P})+C_{\text {int }} J_{H}\left(w_{H}, \mathcal{F}\right)\right)\left|v_{h}\right|_{1}+2 C_{\text {stab }}\left|u_{H}-w_{H}\right|_{1}\left|v_{h}\right|_{1},
\end{aligned}
$$

where $C_{\text {stab }}$ is the $H_{0}^{1}$-stability constant of the weighted Clément operator. We conclude by setting $C_{1}=$ $\max \left(C_{\text {orth }}, C_{\text {int }}, 2 C_{\text {stab }}\right)$.

The next lemma concerns lower bounds of the error. Whereas the global bound has a standard form, the local bound is different from the one commonly used for convergence proofs, since the inner node property is not assumed here.

Lemma 3.2 (lower bounds). There exists a constant $C_{2}>0$ depending only on the minimum angle of $h_{0}$ such that for all $v_{H} \in V_{H}$

$$
J_{H}^{2}\left(v_{H}\right) \leq C_{2}\left(\left|u-v_{H}\right|_{1}^{2}+\operatorname{osc}_{H}^{2}\right) .
$$

There exists a constant $C_{4}>0$ depending only on the minimum angle of $h_{0}$ such that for $\mathcal{F} \subset \mathcal{E}_{H}, h=$ $\mathcal{R}_{\text {loc }}(H, \mathcal{F})$ and arbitrary $\delta>0$

$$
J_{h}^{2}\left(v_{h}\right) \leq(1+\delta) J_{H}^{2}\left(v_{H}\right)-\frac{1+\delta}{2} J_{H}^{2}\left(v_{H}, \mathcal{F}\right)+C_{4}(1+1 / \delta)\left|v_{h}-v_{H}\right|_{1}^{2} \quad \forall v_{h} \in V_{h}, v_{H} \in V_{H} .
$$

Proof. The global local bound (3.4) is obtained using the standard techniques of [20] as follows. First we recall that for a given triangle $K \in \mathcal{K}_{H}$ and given edge $E \in \mathcal{E}_{H}$ there exist a cubic bubble function $b_{K} \in H_{0}^{1}(K)$ and a quadratic bubble function $b_{E} \in H_{0}^{1}\left(\omega_{E}\right)$, where $\omega_{E}$ denotes the union of the neighboring cells of $E$. Taking the test function $w_{E}:=\left[\frac{\partial v_{H}}{\partial n}\right] b_{E}$ we have with positive constants $C, c>0$ and $f_{K}=\pi_{K} f$ that

$$
\begin{aligned}
c\left\|\left[\frac{\partial v_{H}}{\partial n}\right]\right\|_{E}^{2} & \leq \int_{E}\left[\frac{\partial v_{H}}{\partial n}\right] w_{E} \mathrm{~d} s=\int_{\omega_{E}} \nabla v_{H} \nabla w_{E} \mathrm{~d} x=\int_{\omega_{E}} \nabla\left(v_{H}-u\right) \nabla w_{E} \mathrm{~d} x+\int_{\omega_{E}} f w_{E} \mathrm{~d} x \\
& =\int_{\omega_{E}} \nabla\left(v_{H}-u\right) \nabla w_{E} \mathrm{~d} x+\sum_{K \subset \omega_{E}} \int_{\omega_{E}} f_{K} w_{E} \mathrm{~d} x+\int_{\omega_{E}}\left(f-f_{K}\right) w_{E} \mathrm{~d} x \\
& \leq C\left(|E|^{-1 / 2}\left|u-v_{H}\right|_{1, \omega_{E}}+|E|^{1 / 2} \sum_{K \subset \omega_{E}}\left(\left\|f_{K}\right\|_{K}+\left\|f-f_{K}\right\|_{K}\right)\right)\left\|\left[\frac{\partial v_{H}}{\partial n}\right]\right\|_{E},
\end{aligned}
$$


which yields

$$
|E|\left\|\left[\frac{\partial v_{H}}{\partial n}\right]\right\|_{E}^{2} \leq C\left(\left|u-v_{H}\right|_{1, \omega_{E}}^{2}+\sum_{K \subset \omega_{E}}|K|\left\|f-f_{K}\right\|_{K}^{2}+\sum_{K \subset \omega_{E}}|K|\left\|f_{K}\right\|_{K}^{2}\right) .
$$

In order to estimate the last term in (3.6), we choose the test function $w_{K}:=f_{K} b_{K}$, yielding

$$
\int_{K} \nabla\left(u-v_{H}\right) \cdot \nabla w_{K} \mathrm{~d} x=\int_{K} f w_{K} \mathrm{~d} x=\int_{K} f_{K} w_{K} \mathrm{~d} x+\int_{K}\left(f-f_{K}\right) w_{K} \mathrm{~d} x,
$$

from which we deduce that

$$
c\left\|f_{K}\right\|_{K}^{2} \leq C\left(|K|^{-1 / 2}\left|u-v_{H}\right|_{1, K}+\left\|f-f_{K}\right\|_{K}\right)\left\|f_{K}\right\|_{K},
$$

and therefore

$$
|K|\left\|f_{K}\right\|_{K}^{2} \leq C\left(\left|u-v_{H}\right|_{1, K}^{2}+|K||| f-f_{K} \|_{K}^{2}\right) .
$$

Combining the last inequality with (3.6) and making use of the estimate

$$
\sum_{K \in \mathcal{K}_{H}}|K|\left\|f-f_{K}\right\|_{K}^{2} \leq C \operatorname{osc}_{H}^{2}
$$

gives (3.1). The bound (3.7) follows from the fact that for a node $z \in \mathcal{N}_{H}$, it holds $\sum_{K \subset \omega_{z}}\left\|f-f_{K}\right\|_{K}^{2} \leq$ $\sum_{K \subset \omega_{z}}\left\|f-\pi_{z} f\right\|_{K}^{2}$ and that the number of cells sharing a node is bounded for the family of meshes.

Next we prove (3.5), following [10]. We divide the edges created by the local refinement into three sets. The first set consists of newly created edges in the interior of a coarse cell. For such an edge $E$ we have that

$$
|E| \int_{E}\left[\frac{\partial v_{h}}{\partial n}\right]^{2} \mathrm{~d} s=|E| \int_{E}\left[\frac{\partial\left(v_{h}-v_{H}\right)}{\partial n}\right]^{2} \mathrm{~d} s \leq C\left|v_{h}-v_{H}\right|_{1, \omega_{E}}^{2} .
$$

The second set consists of edges obtained from dividing a coarse edge $E$ into two new edges, $E=E_{1} \cup E_{2}$. For a coarse edge from this set, we have by Young's inequality

$$
\begin{aligned}
\left|E_{1}\right| \int_{E_{1}}\left[\frac{\partial v_{h}}{\partial n}\right]^{2} \mathrm{~d} s & +\left|E_{2}\right| \int_{E_{2}}\left[\frac{\partial v_{h}}{\partial n}\right]^{2} \mathrm{~d} s \\
& \leq(1+\delta) \frac{|E|}{2} \int_{E}\left[\frac{\partial v_{H}}{\partial n}\right]^{2} \mathrm{~d} s+(1+1 / \delta) \frac{|E|}{2} \int_{E}\left[\frac{\partial\left(v_{h}-v_{H}\right)}{\partial n}\right]^{2} \mathrm{~d} s \\
& \leq\left(1+\delta-\frac{1+\delta}{2}\right)|E| \int_{E}\left[\frac{\partial v_{H}}{\partial n}\right]^{2} \mathrm{~d} s+(1+1 / \delta) \frac{|E|}{2} \int_{E}\left[\frac{\partial\left(v_{h}-v_{H}\right)}{\partial n}\right]^{2} \mathrm{~d} s
\end{aligned}
$$

yielding (3.5) in this case.

The last set is the set of unrefined edges. Here we have

$$
\begin{aligned}
|E| \int_{E}\left[\frac{\partial v_{h}}{\partial n}\right]^{2} \mathrm{~d} s & \leq(1+\delta)|E| \int_{E}\left[\frac{\partial v_{H}}{\partial n}\right]^{2} \mathrm{~d} s+(1+1 / \delta)|E| \int_{E}\left[\frac{\partial\left(v_{h}-v_{H}\right)}{\partial n}\right]^{2} \mathrm{~d} s \\
& \leq(1+\delta)|E| \int_{E}\left[\frac{\partial v_{H}}{\partial n}\right]^{2} \mathrm{~d} s+C(1+1 / \delta)\left|v_{h}-v_{H}\right|_{1, \omega_{E}}^{2} .
\end{aligned}
$$

This completes the proof. 


\section{Convergence proof}

We prove error reduction with respect to the following error measure:

$$
e(h, m):=\left|u-u_{h}^{m}\right|_{1}^{2}+\beta_{1} \operatorname{osc}_{h}^{2}+\beta_{2} J_{h}^{2}\left(u_{h}^{m}\right)
$$

for some constants $\beta_{1}>0$ and $\beta_{2}>0$.

Theorem 4.1. Assume that $\zeta$ satisfies (2.3). Let $\left\{h_{k}\right\}_{k>0}$ be a sequence of meshes generated by algorithm $\mathcal{A} \mathcal{F} \mathcal{E} \mathcal{M}$ and let $\left\{u_{h_{k}}^{m_{k}}\right\}_{k \geq 0}$ be the corresponding sequence of finite element solutions. Suppose that

$$
0 \leq \alpha<\frac{\theta^{2}}{64 C_{4} C_{m g}}
$$

then there exist constants $\beta_{1}>0, \beta_{2}>0$, and $\rho<1$ such that for all $k=1,2, \ldots$

$$
e\left(h_{k+1}, m_{k+1}\right) \leq \rho e\left(h_{k}, m_{k}\right) .
$$

Remark 4.2. For the convergence result of Theorem 4.1, the parameters $\gamma, \theta<1$, and $\sigma<1$ can be chosen arbitrarily.

Proof. We set $\alpha^{\prime}:=\alpha C_{m g}$.

First we set $u_{h}:=u_{h_{k+1}}, u_{H}:=u_{h_{k}}, u_{h}^{m}=u_{h_{k+1}}^{m_{k+1}}, u_{H}^{l}=u_{h_{k}}^{m_{k}}, J_{h}=J_{h}\left(u_{h}^{m}\right), J_{H}=J_{H}\left(u_{H}^{l}\right), J_{H}(\mathcal{F}):=$ $J_{H}\left(u_{H}^{l}, \mathcal{F}\right)$ and similarly for the other involved quantities. We consider the two cases of the algorithm separately.

Let us start with the first case. We make use of the Galerkin property of the finite element discretization. Since for arbitrary $\xi>0$ we have

$$
\begin{aligned}
\left\langle\nabla\left(u-u_{h}^{m}\right), \nabla\left(u_{h}^{m}-u_{H}^{l}\right)\right\rangle & =\left\langle\nabla\left(u_{h}-u_{h}^{m}\right), \nabla\left(u_{h}^{m}-u_{H}^{l}\right)\right\rangle \\
& \leq(1 / 2 \xi)\left|u_{h}-u_{h}^{m}\right|_{1}^{2}+\xi / 2\left|u_{h}^{m}-u_{H}^{l}\right|_{1}^{2},
\end{aligned}
$$

we find by means of the stopping criterion that

$$
\begin{aligned}
\left|u-u_{h}^{m}\right|_{1}^{2} & =\left|u-u_{H}^{l}\right|_{1}^{2}-\left|u_{h}^{m}-u_{H}^{l}\right|_{1}^{2}-2\left\langle\nabla\left(u-u_{h}^{m}\right), \nabla\left(u_{h}^{m}-u_{H}^{l}\right)\right\rangle \\
& \leq\left|u-u_{H}^{l}\right|_{1}^{2}-(1-\xi)\left|u_{h}^{m}-u_{H}^{l}\right|_{1}^{2}+\frac{\alpha^{\prime}}{\xi} J_{h}^{2} .
\end{aligned}
$$

Combining the last inequality with (3.5) and introducing $\beta_{2}^{\prime}:=\beta_{2}+\alpha^{\prime} / \xi$ we obtain

$$
\begin{aligned}
\left|u-u_{h}^{m}\right|_{1}^{2}+\beta_{2} J_{h}^{2} \leq & \left|u-u_{H}^{l}\right|_{1}^{2}-(1-\xi)\left|u_{h}^{m}-u_{H}^{l}\right|_{1}^{2}+\beta_{2}^{\prime} J_{h}^{2} \\
\leq & \left|u-u_{H}^{l}\right|_{1}^{2}+\left(\beta_{2}^{\prime} C_{4}(1+1 / \delta)-1+\xi\right)\left|u_{h}^{m}-u_{H}^{l}\right|_{1}^{2} \\
& +\beta_{2}^{\prime}\left((1+\delta) J_{H}^{2}-\frac{1+\delta}{2} J_{H}^{2}(\mathcal{F})\right),
\end{aligned}
$$

which leads to

$$
\begin{aligned}
e(h, m) \leq & \left|u-u_{H}^{l}\right|_{1}^{2}+\left(\beta_{2}^{\prime} C_{4}(1+1 / \delta)-1+\xi\right)\left|u_{h}^{m}-u_{H}^{l}\right|_{1}^{2}+\beta_{1} \operatorname{osc}_{h}^{2} \\
& +\beta_{2}^{\prime}\left((1+\delta) J_{H}^{2}-\frac{1+\delta}{2} J_{H}^{2}(\mathcal{F})\right)
\end{aligned}
$$

Imposing the condition

$$
\left(\beta_{2}+\frac{\alpha^{\prime}}{\xi}\right) C_{4}(1+1 / \delta)-1+\xi \leq 0,
$$


and using the refinement criterion, we have

$$
e(h, m) \leq\left|u-u_{H}^{l}\right|_{1}^{2}+\beta_{1} \operatorname{osc}_{h}^{2}+\beta_{2}^{\prime}\left((1+\delta) J_{H}^{2}-\frac{\theta(1+\delta)}{2} J_{H}^{2}\right) .
$$

We next introduce $\theta^{\prime}=(1+\delta) \theta / 2-\delta$ and parameters $a, b>0$ to be chosen later.

$$
\begin{aligned}
e(h, m) & \leq\left|u-u_{H}^{l}\right|_{1}^{2}+\beta_{1} \operatorname{osc}_{h}^{2}+\beta_{2}^{\prime}\left(1-\theta^{\prime}\right) J_{H}^{2} \\
& \leq\left|u-u_{H}^{l}\right|_{1}^{2}-a \theta^{\prime} \beta_{2}^{\prime} J_{H}^{2}+\beta_{1} \operatorname{osc}_{h}^{2}-(1-a-b) \theta^{\prime} \beta_{2}^{\prime} J_{H}^{2}+\beta_{2}^{\prime}\left(1-b \theta^{\prime}\right) J_{H}^{2} .
\end{aligned}
$$

Using the upper bound we have $\left|u-u_{H}^{l}\right|_{1}^{2} \leq\left(C_{1}+2 \alpha^{\prime}\right)\left(J_{H}^{2}+\operatorname{osc}_{H}^{2}\right)$. Taking into account osc ${ }_{H}^{2} \leq \gamma J_{H}^{2}$ we find

$$
\begin{aligned}
e(h, m) \leq & \left(1-\frac{a \beta_{2}^{\prime} \theta^{\prime}}{C_{1}+2 \alpha^{\prime}}\right)\left|u-u_{H}^{l}\right|_{1}^{2}+\left(\beta_{1}+a \theta^{\prime} \beta_{2}^{\prime}\right) \operatorname{osc}_{H}^{2} \\
& -(1-a-b) \theta^{\prime} \beta_{2}^{\prime} J_{H}^{2}+\beta_{2}^{\prime}\left(1-b \theta^{\prime}\right) J_{H}^{2} \\
\leq & \left(1-\frac{a \beta_{2}^{\prime} \theta^{\prime}}{C_{1}+2 \alpha^{\prime}}\right)\left|u-u_{H}^{l}\right|_{1}^{2}+\beta_{1}\left(1+\theta^{\prime} \frac{\beta_{2}^{\prime}}{\beta_{1}}\left(a-\frac{1-a-b}{\gamma}\right)\right) \operatorname{osc}_{H}^{2} \\
& +\beta_{2}^{\prime}\left(1-b \theta^{\prime}\right) J_{H}^{2} \\
\leq & \rho_{1}\left|u-u_{H}^{l}\right|_{1}^{2}+\rho_{2} \beta_{1} \operatorname{osc}_{H}^{2}+\rho_{3} \beta_{2} J_{H}^{2} \\
\leq & \rho^{1} e(H, l)
\end{aligned}
$$

with $\rho^{1}=\max \left(\rho_{1}, \rho_{2}, \rho_{3}\right)$ and

$$
\rho_{1}=1-a \beta_{2}^{\prime} \theta^{\prime}\left(C_{1}+2 \alpha^{\prime}\right)^{-1}, \rho_{2}=1+\theta^{\prime} \frac{\beta_{2}^{\prime}}{\beta_{1}}\left(a-\frac{1-a-b}{\gamma}\right), \rho_{3}=\left(\beta_{2}^{\prime} / \beta_{2}\right)\left(1-\theta^{\prime} b\right) .
$$

We now show that under the condition (4.2) we have $0<\rho_{i}<1$ for $i=1,2,3$.

First we notice that (4.2) implies, since $1-x<\sqrt{1-x} \leq 1-x / 2-x^{2} / 8$ for $0<x<1$ (which we apply with $x=\theta / 2)$,

$$
\alpha^{\prime}<\frac{\theta^{2}}{64 C_{4}} \leq \frac{1-\theta / 4-\sqrt{1-\theta / 2}}{2 C_{4}}<\frac{1-\theta / 4-(1-\theta / 2)}{2 C_{4}}=\frac{\theta}{8 C_{4}} .
$$

Next we consequently choose the parameters $\delta, b$, and $\beta_{2}$. First consider the quadratic equation

$$
\delta^{2}+A \delta+B=0, \quad A=\frac{4 \alpha^{\prime} C_{4}-\theta / 2}{1-\theta / 2}, \quad B=\frac{4 \alpha^{\prime} C_{4}}{1-\theta / 2} .
$$

By (4.6) we have $A<0$ and $A^{2} / 4-B>0$. Therefore (4.7) has a positive root and this implies existence of $\delta^{*}>0$ such that

$$
(1-\theta / 2) \delta^{* 2}+\left(4 \alpha^{\prime} C_{4}-\theta / 2\right) \delta^{*}<-4 \alpha^{\prime} C_{4}
$$

We choose $\delta<\min \left(\delta^{*}, \frac{\theta / 2}{1-\theta / 2}\right)$. (4.8) implies

$$
\frac{\alpha^{\prime} C_{4}(1+1 / \delta)}{\theta^{\prime}}=\frac{\alpha^{\prime} C_{4}(1+1 / \delta)}{\theta / 2-\delta(1-\theta / 2)}<\frac{1}{4}
$$

Due to (4.9) the quadratic equation

$$
x-x^{2}=\frac{\alpha^{\prime} C_{4}(1+1 / \delta)}{\theta^{\prime}}
$$


has two roots satisfying $0<\xi_{1}<\xi_{2}<1$. Choose $\xi_{1}<\xi<\xi_{2}$, such that

$$
\xi-\xi^{2}>\frac{\alpha^{\prime} C_{4}(1+1 / \delta)}{\theta^{\prime}}
$$

Direct manipulation leads to

$$
\frac{1-\theta^{\prime}}{\theta^{\prime}} \frac{\alpha^{\prime}}{\xi}<\frac{1-\xi}{C_{4}(1+1 / \delta)}-\frac{\alpha^{\prime}}{\xi} .
$$

We can therefore choose $\beta_{2}>0$ such that

$$
\frac{1-\theta^{\prime}}{\theta^{\prime}} \frac{\alpha^{\prime}}{\xi}<\beta_{2}<\frac{1-\xi}{C_{4}(1+1 / \delta)}-\frac{\alpha^{\prime}}{\xi}
$$

The left inequality in (4.13) leads to

$$
\theta^{\prime}>\frac{\alpha^{\prime} / \xi}{\beta_{2}+\alpha^{\prime} / \xi}=1-\frac{\beta_{2}}{\beta_{2}+\alpha^{\prime} / \xi}=1-\frac{\beta_{2}}{\beta_{2}^{\prime}} .
$$

This allows us to choose $b$ such that

which implies

$$
1>b>\left(1-\frac{\beta_{2}}{\beta_{2}^{\prime}}\right) / \theta^{\prime}
$$

$$
\rho_{3}=\frac{\beta_{2}^{\prime}}{\beta_{2}}\left(1-b \theta^{\prime}\right)<1 \text {. }
$$

The right inequality in (4.13) leads to

$$
\beta_{2}^{\prime} C_{4}(1+1 / \delta)+\xi \leq 1
$$

which is (4.5).

It remains to choose $a<\frac{1-b}{1+\gamma}$ to obtain $\rho_{2}<1$. Since for the indicated choices, it holds $\rho_{1}<1$, we have completed the proof in the first case. Notice that $\beta_{1}>0$ is arbitrary up to now.

Now we consider the second case of the algorithm. We have the following property concerning the oscillation term involving a constant $0<\mu<1$ :

$$
\operatorname{Osc}_{H}^{2}-\operatorname{osc}_{h}^{2} \geq \mu \operatorname{osc}_{H}^{2}(\mathcal{P})
$$

which follows from the facts that the number of triangles containing a given node $z \in \mathcal{N}_{h}$ is bounded and that the marked cells are at least bisected once, see [18] for more details.

The inequality (4.17) implies $\operatorname{osc}_{h}^{2} \leq(1-\mu \sigma) \operatorname{osc}_{H}^{2}$. With the same choice of $\beta_{2}, \xi$, and $\delta$ as before, it follows from (4.8) under the condition (4.5) that:

$$
\begin{aligned}
e(h, m) \leq & \left|u-u_{H}^{l}\right|_{1}^{2}+\left(\beta_{2}^{\prime} C_{4}(1+1 / \delta)-1+\xi\right)\left|u_{h}^{m}-u_{H}^{l}\right|_{1}^{2}+\beta_{1} \operatorname{osc}_{h}^{2} \\
& +\beta_{2}^{\prime}\left((1+\delta) J_{H}^{2}-\frac{1+\delta}{2} J_{H}^{2}(\mathcal{F})\right) \\
\leq & \left|u-u_{H}^{l}\right|_{1}^{2}+\beta_{1} \operatorname{osc}_{h}^{2}+\beta_{2}^{\prime}(1+\delta) J_{H}^{2} \\
\leq & \left|u-u_{H}^{l}\right|_{1}^{2}-a \beta_{1} \mu \sigma \operatorname{osc}_{H}^{2}+\beta_{1}\left(\operatorname{osc}_{H}^{2}-(1-a-b) \mu \sigma \operatorname{osc}_{H}^{2}\right) \\
& +\beta_{2}^{\prime}(1+\delta) J_{H}^{2}-b \beta_{1} \mu \sigma \operatorname{osc}_{H}^{2} \\
\leq & \left(1-a \frac{\beta_{1} \mu \sigma \gamma}{C_{1}(1+\gamma)+2 \alpha^{\prime}}\right)\left|u-u_{H}^{l}\right|_{1}^{2}+\beta_{1}(1-(1-a-b) \mu \sigma) \operatorname{osc}_{H}^{2} \\
& +\beta_{2}\left(\left(1+\frac{\alpha^{\prime}}{\beta_{2} \xi}\right)(1+\delta)-b \frac{\beta_{1}}{\beta_{2}} \gamma \mu \sigma\right) J_{H}^{2} \\
\leq & \rho^{(2)} e(H, l) .
\end{aligned}
$$


We choose $a, b$ such that $1-a-b>0$ and $\beta_{1}$ such that

$$
\beta_{1}>\frac{\beta_{2}^{\prime}(1+\delta)}{b \gamma \mu \sigma}
$$

It follows that $0<\rho^{(2)}<1$. We conclude the proof by setting $\rho=\max \left(\rho^{1}, \rho^{(2)}\right)$.

\section{Complexity estimate}

In order to express the optimal complexity, we introduce some notation from nonlinear approximation theory, developed in $[6,13]$. Let $\mathcal{H}_{N}$ be the set of all triangulations $h$ which satisfy $N_{h} \leq N$.

Next we define the approximation class

$$
\mathcal{W}^{s}:=\left\{(u, f) \in\left(H_{0}^{1}(\Omega), L^{2}(\Omega)\right):\|(u, f)\|_{\mathcal{W}^{s}}<+\infty\right\}
$$

with

$$
\|(u, f)\|_{\mathcal{W}^{s}}:=\sup _{N \geq N_{0}} N^{s} \inf _{h \in H_{N}}\left(\left|u-u_{h}\right|_{1}^{2}+\operatorname{osc}_{h}^{2}\right) .
$$

We say that an adaptive finite element method realizes optimal convergence rates if, whenever $(u, f) \in \mathcal{W}^{s}$, it produces a triangulation $h_{k}$ with dimension $N_{k}$ and corresponding approximation $u_{k}$ such that

$$
\left|u-u_{k}\right|_{1}^{2} \leq C N_{k}^{-s}
$$

Theorem 5.1. Suppose $(f, u) \in \mathcal{W}^{s}$. Let $\left\{h_{k}\right\}_{k \geq 0}$ be a sequence of meshes generated by algorithm $\mathcal{A} \mathcal{F} \mathcal{E} \mathcal{M}$ and let $\left\{V_{k}\right\}_{k \geq 0}$ and $\left\{u_{k}\right\}_{k \geq 0}$ be the corresponding sequences of finite element spaces and solutions. Let $\varepsilon_{k}:=$ $\left|u-u_{k}^{m_{k}}\right|_{1}^{2}+\operatorname{osc}_{k}^{2}$ and $N_{k}=\operatorname{dim}\left(V_{k}\right)$. Assuming the parameters $\gamma$ and $\theta$ to satisfy

$$
0<\gamma<1 /\left(C_{2}\left(1+C_{1}\right)\right), \quad 0<\theta<\frac{1-\gamma C_{2}\left(1+C_{1}\right)}{C_{2}\left(C_{1}+C_{m g} \alpha\right)}
$$

we have the following estimate on the complexity of the algorithm: there exists a constant $C$ such that

$$
N_{k} \leq C \varepsilon_{k}^{-1 / s}
$$

Remark 5.2. In case of exact solution, $\alpha=0$, and zero data oscillation $\operatorname{osc}_{h}=0$ (and consequently $\gamma=0$ ), we get the condition $\theta<1 /\left(C_{1} C_{2}\right)$. This condition states that the percentage of marked cells should not be larger than the inverse of the efficiency index of the estimator $C_{1} C_{2}$. Such a condition seems natural, since $\theta=1$ corresponds to uniform refinement.

Proof. We use the same notation as in the convergence proof. As before, we consider the two cases separately.

First let $\operatorname{osc}_{H}^{2} \leq \gamma J_{H}^{2}$. From our regularity assumption we have existence of a mesh $h^{*} \in \mathcal{H}$ such that for $\lambda>0$ to be chosen below

and

$$
\left|u-u_{h^{*}}\right|_{1}^{2}+\operatorname{osc}_{h^{*}}^{2} \leq \lambda\left(\left|u-u_{H}^{l}\right|_{1}^{2}+\operatorname{osc}_{H}^{2}\right)
$$

$$
N_{h^{*}} \leq C\left(\left|u-u_{H}^{l}\right|_{1}^{2}+\operatorname{osc}_{H}^{2}\right)^{-1 / s} .
$$

Following the proof of Stevenson [19] (proof of Lem. 5.2), we can suppose that $h^{*}$ is a refinement of $H$, if we replace (5.6) by:

$$
N_{h^{*}}-N_{H} \leq\left(\left|u-u_{H}^{l}\right|_{1}^{2}+\operatorname{osc}_{H}^{2}\right)^{-1 / s} .
$$

Let $\mathcal{F}^{*} \subset \mathcal{E}_{H}$ be the set of refined edges and let $\mathcal{P}^{*}$ be the set of corresponding nodes. We now prove that

$$
J_{H}^{2}\left(\mathcal{F}^{*}\right) \geq \theta J_{H}^{2} .
$$


From (3.2) and the stopping criterion we have, since $\left|u-u_{h^{*}}\right|_{1}^{2}=\left|u-u_{H}^{l}\right|_{1}^{2}-\left|u_{H}^{l}-u_{h^{*}}\right|_{1}^{2}$,

$$
\begin{aligned}
\left(C_{1}+\alpha^{\prime}\right) J_{H}^{2}\left(\mathcal{F}^{*}\right) & \geq\left|u_{h^{*}}-u_{H}^{l}\right|_{1}^{2}-C_{1} \operatorname{osc}_{H}^{2}\left(\mathcal{P}^{*}\right)=\left|u-u_{H}^{l}\right|_{1}^{2}-\left|u-u_{h^{*}}\right|_{1}^{2}-C_{1} \operatorname{osc}_{H}^{2}\left(\mathcal{P}^{*}\right) \\
& \geq\left|u-u_{H}^{l}\right|_{1}^{2}-\lambda\left(\left|u-u_{H}^{l}\right|_{1}^{2}+\operatorname{osc}_{H}^{2}\right)+\operatorname{osc}_{h^{*}}-C_{1} \operatorname{osc}_{H}^{2}\left(\mathcal{P}^{*}\right) \\
& \geq(1-\lambda)\left(\left|u-u_{H}^{l}\right|_{1}^{2}+\operatorname{osc}_{H}^{2}\right)-\operatorname{osc}_{H}^{2}-C_{1} \operatorname{osc}_{H}^{2}\left(\mathcal{P}^{*}\right) \\
& \geq \frac{1-\lambda}{C_{2}} J_{H}^{2}-\left(1+C_{1}\right) \operatorname{osc}_{H}^{2} \\
& \geq\left(\frac{1-\lambda}{C_{2}}-\gamma\left(1+C_{1}\right)\right) J_{H}^{2} .
\end{aligned}
$$

Choosing $\lambda=1-C_{2}\left(C_{1}+\alpha^{\prime}\right) \theta-\gamma C_{2}\left(1+C_{1}\right)$, which is positive by assumption, we obtain the desired bound (5.8).

Since $\mathcal{F}$ is chosen to be the set with minimal cardinality satisfying the bound (5.8), we find that

$$
\# \mathcal{F}_{k} \leq \# \mathcal{F}^{*} \leq N_{h^{*}}-N_{k} \leq \varepsilon_{k}^{-1 / s}
$$

Now we consider the second case. Similarly as before, there exists a mesh $h^{*}$ such that with $\lambda$ to be chosen below

$$
\left|u-u_{h^{*}}\right|_{1}^{2}+\operatorname{osc}_{h^{*}}^{2} \leq \lambda\left(\left|u-u_{H}^{l}\right|_{1}^{2}+\operatorname{osc}_{H}^{2}\right)
$$

and

$$
N_{h^{*}}-N_{h} \leq C\left(\left|u-u_{H}^{l}\right|_{1}^{2}+\operatorname{osc}_{H}^{2}\right)^{-1 / s} .
$$

It follows from (5.10) and (3.1) that

$$
\begin{aligned}
\operatorname{osc}_{H}^{2}-\operatorname{osc}_{H}^{2}\left(\mathcal{P}^{*}\right) & \leq \operatorname{osc}_{h^{*}}^{2} \leq \lambda\left(\left|u-u_{H}^{l}\right|_{1}^{2}+\operatorname{osc}_{H}^{2}\right) \\
& \leq \lambda\left(\left(C_{1}+\alpha^{\prime}\right)\left(J_{H}^{2}+\operatorname{osc}_{H}^{2}\right)+\operatorname{osc}_{H}^{2}\right) \\
& \leq \lambda\left(1+\left(C_{1}+\alpha^{\prime}\right)\left(1+\frac{1}{\gamma}\right)\right) \operatorname{osc}_{H}^{2} .
\end{aligned}
$$

From this we obtain

$$
\operatorname{osc}_{H}^{2}\left(\mathcal{P}^{*}\right) \geq\left(1-\lambda\left(1+\left(C_{1}+\alpha^{\prime}\right)\left(1+\frac{1}{\gamma}\right)\right)\right) \operatorname{osc}_{H}^{2}
$$

We choose $\lambda=\frac{1-\sigma}{1+\left(C_{1}+\alpha^{\prime}\right)\left(1+\frac{1}{\gamma}\right)}$ to obtain

$$
\operatorname{osc}_{H}^{2}\left(\mathcal{P}^{*}\right) \geq \sigma \operatorname{osc}_{H}^{2}
$$

With the same argument as before we get (5.9).

Let now $e_{l}:=\left|u-u_{h_{l}}^{m_{l}}\right|_{1}^{2}+\beta_{1} \operatorname{osc}_{h_{l}}^{2}+\beta_{2} J_{h_{l}}^{2}\left(u_{h_{l}}^{m_{l}}\right)$. From Theorem 4.1 we know that with a constant $0<\rho<1$ there holds

$$
e_{k} \leq \rho^{k-l} e_{l}, \quad k \geq l \geq 0 .
$$

Due to the definitions and the first lower bound (3.4) of Lemma $3.2 e_{k}$ and $\varepsilon_{k}$ are equivalent. Then we also have with a constant $C$ depending on $\beta_{1}, \beta_{2}$ that

$$
\varepsilon_{k} \leq C \rho^{k-l} \varepsilon_{l}, \quad k \geq l \geq 0 .
$$


The estimate (5.13) together with Assumption 2.1 imply

$$
\begin{aligned}
N_{k+1}-N_{0} & \leq C \sum_{l=0}^{k} \# \mathcal{F}_{k} \leq C \sum_{l=0}^{k} \varepsilon_{l}^{-1 / s} \\
& \leq C \varepsilon_{k}^{-1 / s} \sum_{l=0}^{k}\left(\varepsilon_{k} / \varepsilon_{l}\right)^{1 / s} \leq C \varepsilon_{k}^{-1 / s}\left(\sum_{l=0}^{k} \rho^{(k-l) / s}\right) \leq \frac{C}{1-\rho^{1 / s}} \varepsilon_{k}^{-1 / s}
\end{aligned}
$$

This concludes the proof.

By Theorem 5.1, the number of unknowns is asymptotically optimal. The convergence analysis of multigrid on the type of meshes considered here, see [7,21], can be used to check that the stopping criterion of the multigrid iteration can be fulfilled with linear work count (supposing we use the nested algorithm). We note that it also has already been discussed in the literature, see [14], how the logarithmic factor required in the complete sorting of the estimator can be avoided.

Corollary 5.3. The algorithm $\mathcal{A F \mathcal { F }}$ can be implemented with optimal work count in the sense that for a given accuracy $\varepsilon>0$, the algorithm provides a discrete solution $u_{h}$ satisfying $\left|u-u_{h}\right|_{1} \leq \varepsilon$ with a number of operations proportional to $\varepsilon^{-1 / 2 s}$.

\section{Stopping CRITERION}

One way to obtain an a posteriori error estimator satisfying (2.3) is to follow the idea of [4].

The hierarchical refinement leads to a sequence of nested spaces $V_{1} \subset \ldots \subset V_{l} \subset \ldots \subset V_{L}$ with $V_{L}=V_{h}$. Let the discrete operators $A_{l}: V_{l} \rightarrow V_{l}$ be defined by $\left\langle A_{l} u_{l}, v_{l}\right\rangle=\left\langle\nabla u_{l}, \nabla v_{l}\right\rangle$ for all $v_{l} \in V_{l}$ and $Q_{l}: L^{2}(\Omega) \rightarrow V_{l}$ be the $L^{2}(\Omega)$-projection.

Let $u_{h}^{m}$ be the approximate solution of the multigrid iteration after $m$ iterations. We define with $h_{l} \approx 2^{-l}$

$$
\zeta_{h}\left(u_{h}^{m}\right):=\sum_{l=1}^{L} h_{l}\left\|\left(Q_{l}-Q_{l-1}\right) R^{m}\right\|+\left\|Q_{0} R^{m}\right\|, \quad R^{m}:=Q_{h} f-A_{h} u_{h}^{m} .
$$

Using the relation $Q_{l} A_{h}=A_{l} P_{l}$ where $P_{l}$ is the Ritz-projection on $V_{l}$, it can be shown that the residual terms appearing in the definition of (6.1) can be related to the residuals of the multigrid iteration, see [4]. As compared to the estimator proposed there, (6.1) seems to be more appropriate on locally refined meshes. In order to prove that (2.3) is satisfied, let $w:=u_{h}-u_{h}^{m}$. We then have

$$
\begin{aligned}
\left|u_{h}-u_{h}^{m}\right|_{1}^{2} & =\left\langle\nabla\left(u_{h}-u_{h}^{m}\right), \nabla w\right\rangle=\left\langle Q_{h} f-A_{h} u_{h}^{m}, w\right\rangle \\
& =\sum_{l=1}^{L}\left\langle R^{m},\left(Q_{l}-Q_{l-1}\right) w\right\rangle+\left\langle R^{m}, Q_{0} w\right\rangle \\
& =\sum_{l=1}^{L}\left\langle\left(Q_{l}-Q_{l-1}\right) R^{m}, w\right\rangle+\left\langle Q_{0} R^{m}, w\right\rangle \\
& \leq C\left(\sum_{l=1}^{L} h_{l}\left\|\left(Q_{l}-Q_{l-1}\right) R^{m}\right\|+\left\|Q_{0} R^{m}\right\|\right)|w|_{1}
\end{aligned}
$$

by the use of the Clément interpolation and the Poincaré inequality.

Another way to derive such an a posteriori error estimator, which might also be valid for other iterative methods, is given next. Suppose that an a priori estimate of the form

$$
\left|u-u_{h}^{m+1}\right|_{1} \leq \rho_{m g}\left|u-u_{h}^{m}\right|_{1}
$$





FIGURE 1. Refinement creating a hanging node and a hanging nodes and corresponding conforming refinement for triangles.
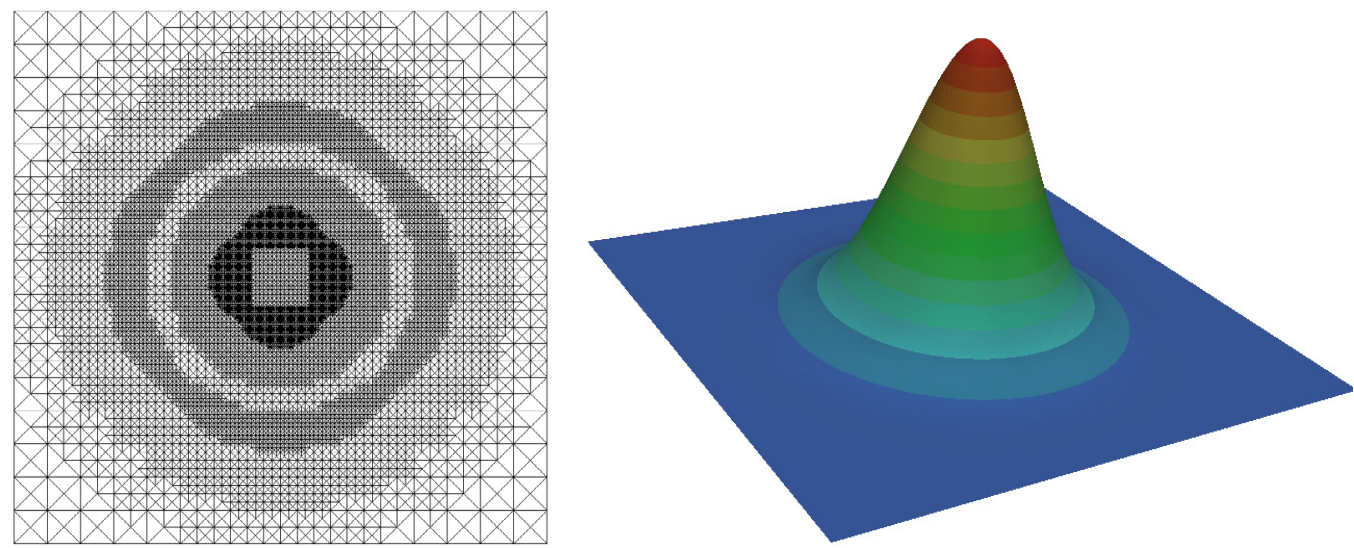

Figure 2. Typical mesh and plot of exact solution for Example 1.

with $\rho_{m g}<1$ holds. Then we define a simple estimator by

$$
\zeta_{h}\left(u_{h}^{m}\right):=\frac{\rho_{m g}}{1-\rho_{m g}}\left|u_{h}^{m+1}-u_{h}^{m}\right|_{1} .
$$

Notice that (6.3) is easy to implement, since it is at hand in standard implementations of the multigrid algorithm and other iterative solvers.

\section{Numerical EXPERIMENTS}

We present two numerical experiments. The first one has a smooth solution and is used to examine the behavior of the algorithm with respect to the different involved parameters. It is also used to compare with other algorithms known from the literature. The second example employs a solution which is not in $H^{2}(\Omega)$. It illustrate the complexity estimate by showing that the optimal error decrease can be recovered by our adaptive algorithm.

For both examples we use $\Omega=]-1,1[\times]-1,1[$. The local mesh refinement uses quadrilateral meshes with hanging nodes which are cut into triangles as indicates in Figure 1. A proof of Assumption 1 for this algorithm as well as further details can be found in [3].

In the first case, we use $u(x)=\exp \left(-10|x|^{2}\right)$ and $f(x)=-\Delta u(x)$. We employ inhomogeneous Dirichlet data, the generalization of the estimator is immediate. This example has been treated before in [17]. A typical mesh is shown in Figure 2. 

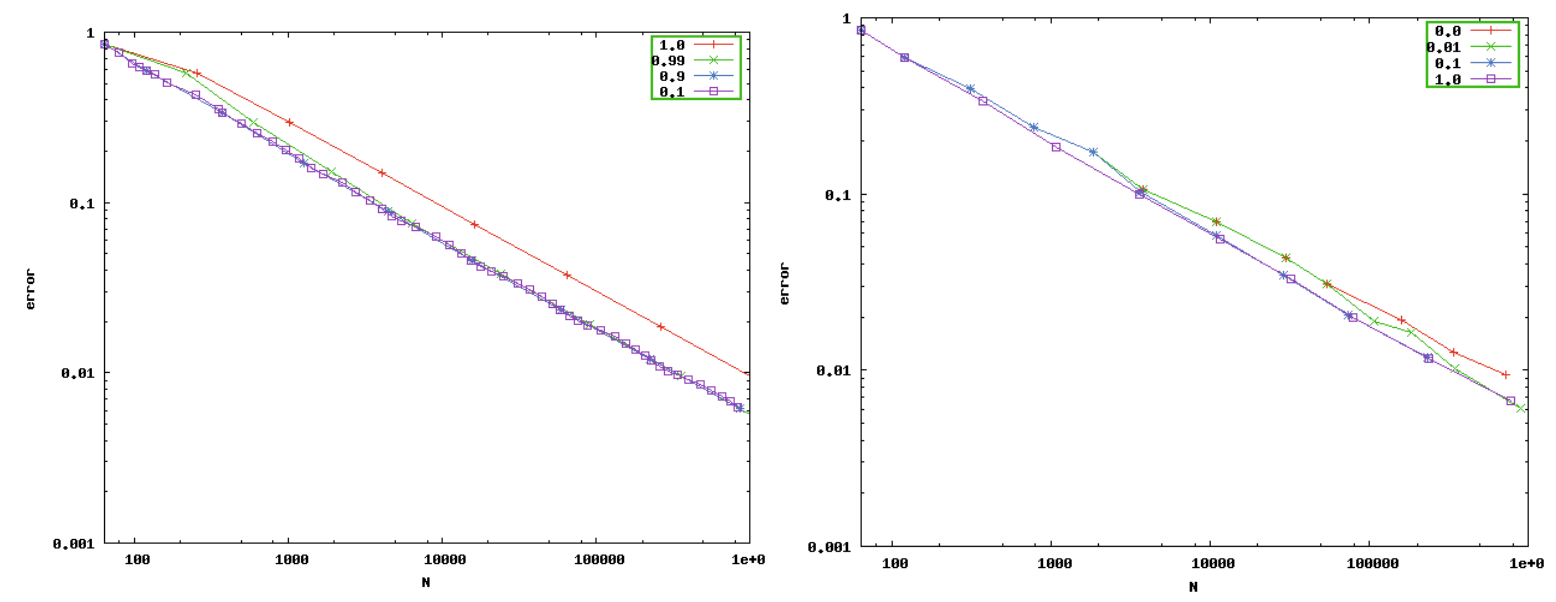

Figure 3. Example 1: Error versus $N$ for different values of $\theta$ (left, $\gamma=0.1$ ) and $\gamma$ (right, $\theta=0.8$ ).

We examine the behaviour of the algorithm with respect to the implied parameters. The important parameters are $\theta$ and $\gamma$. For simplicity, we set throughout $\sigma=\theta$ and $\alpha=0$. The ratio between the true error $e=\left\|\nabla\left(u-u_{h}\right)\right\|$ and the estimator varied between 0.5 and 0.288 in all cases, and between 0.292 and 0.288 for all computations with $N \geq 500$.

The quality of the generated meshes for different values of $\theta$ are shown in the left picture of Figure 3 . Since $u \in H^{2}(\Omega)$, all plots have the optimal slope $s=1 / 2$. We have only represented $\theta=0.9$ and the extreme values $\theta=0.99$ and $\theta=0.1$. It can be noticed that the behaviour is hardly influenced by the value of $\theta$, as long as $\theta<1.0$. In this case (adaptive refinement), there is a gain in the number of unknowns of a factor 2.5-3 as compared to uniform refinement $(\theta=1.0)$, which is explained by a better constant in $e(N) \sim N^{-s}$.

In Table 1 we make a comparison of our algorithm with the MNS algorithm [17]. We have chosen $\gamma=0.1$ and $\theta=0.7$. We denote by $M_{\eta}$ and $M_{\text {osc }}$ the number of marked cells due to the estimator and the oscillation term, respectively. As can bee seen from Table 1, in contrast to the MNS algorithm, the algorithm presented in this article leads to refinement according to the oscillation term only during the first steps. In the following iterations, the refinement is only done according to the jump estimator, which reflects the fact that the oscillations are of higher-order accuracy. Notice that for practical purposes, one would use a larger value of $\theta$ and and even larger value for $\sigma$. The choice of $\theta=0.9$ and $\sigma=0.95$ lead to a reduction of the number of adaptive iterations from 13 to for the same final accuracy in our tests.

Our second numerical example is intended to examine the complexity estimate. We compute the Poisson equation in the L-shaped domain $\Omega=]-1,1[\times]-1,1[\backslash[0,1] \times[0,1]$ and with right-hand side $f=\operatorname{div} g$. The vector $g=\left(g_{1}, g_{2}\right)$ is given by $g_{1}=-1$ if $x>-0.5$ and $g_{1}=1$ otherwise, and $g_{2}=-1$ if $y>-0.5$ and $g_{2}=1$ otherwise. A plot of the solution is shown in Figure 4.

Although the presented theory cannot be applied directly, since $f \notin L^{2}(\Omega)$, we consider this as an interesting example to see, if the optimal slope $s=-1 / 2$ can be reached by the adaptive algorithm. The problem presents a singularity along the lines of discontinuity of $g$, as well as the corner singularity. Both are resolved to a certain extend by the adaptive mesh refinement, as can bee seen from Figure 4.

Finally, we show the decrease of the estimator for different values of $\theta$ in the right plot of Figure 5 . It turns out that slope $s=-1 / 2$ can be recovered for $\theta=0.9$. For $\theta=0.1$ the complexity is not improved, but the number of iterations required to achieve $\eta_{h}<0.1$ is increased from 12 to 74 . The choice of $\theta=0.99$ leads to less favorable behavior, but the optimal slope seems to be recovered when the mesh is fine enough.

The number of nodes is approximately doubled during the adaptive refinement for $\theta \approx 0.9$, which could be expected from isotropic refinement in two dimensions of a one-dimensional singularity. A much better behavior could be expected for this example from anisotropic refinement, but this is out of the present scope. 
TABLE 1. Comparison between MNS algorithm (columns 2-4, from [17]) and the present one (columns 5-7, $\gamma=0.1$ ).

\begin{tabular}{r|r|r|r||r|r|r} 
Iter & $N$ & $M_{\eta}$ & $M_{\mathrm{osc}}$ & $N$ & $M_{\eta}$ & $M_{\mathrm{osc}}$ \\
\hline 0 & 4 & 8 & 0 & 4 & 0 & 4 \\
1 & 64 & 16 & 16 & 16 & 0 & 12 \\
2 & 704 & 56 & 8 & 54 & 0 & 12 \\
3 & 2256 & 80 & 0 & 94 & 0 & 8 \\
4 & 4208 & 96 & 8 & 120 & 0 & 24 \\
5 & 6624 & 112 & 24 & 202 & 0 & 80 \\
6 & 8752 & 344 & 0 & 492 & 0 & 208 \\
7 & 17512 & 432 & 0 & 1224 & 644 & 0 \\
8 & 28368 & 608 & 0 & 3588 & 1796 & 0 \\
9 & 42896 & 768 & 16 & 9396 & 3304 & 0 \\
10 & 60216 & 2192 & 0 & 19466 & 11476 & 0 \\
11 & 113040 & 2296 & 24 & 54388 & 28356 & 0 \\
12 & 160592 & 3816 & 24 & 141144 & 48360 & 0
\end{tabular}
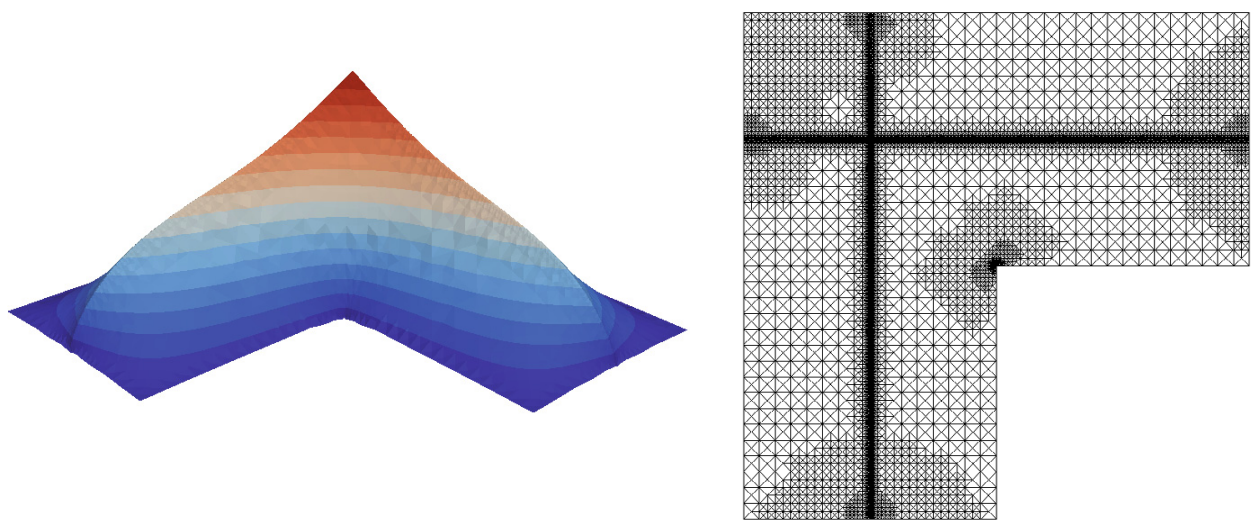

FigurE 4. Typical mesh and plot of exact solution for Example 2.

\section{Conclusion}

We have proposed a new adaptive algorithm for conforming finite elements based on an adaptive marking strategy and an adaptive stopping criterion for iterative solution.

In this article, we have carried out the proofs of geometric convergence of the error and asymptotic complexity of the resulting meshes in the case of lowest-order finite elements in two space dimensions. The generalization to three dimensions seems to be possible.

Numerical examples have been presented in order to compare the adaptive marking strategy with the collective marking known from the literature and in order to illustrate the complexity of the sequence of meshes generated by the adaptive algorithm for different values of the involved parameters. The second example illustrates the gain in adaptivity, but it also suggests the possible improvement by anisotropic refinement. However, the proof of convergence and complexity results for anisotropic adaptive finite element algorithms requires further consideration. 


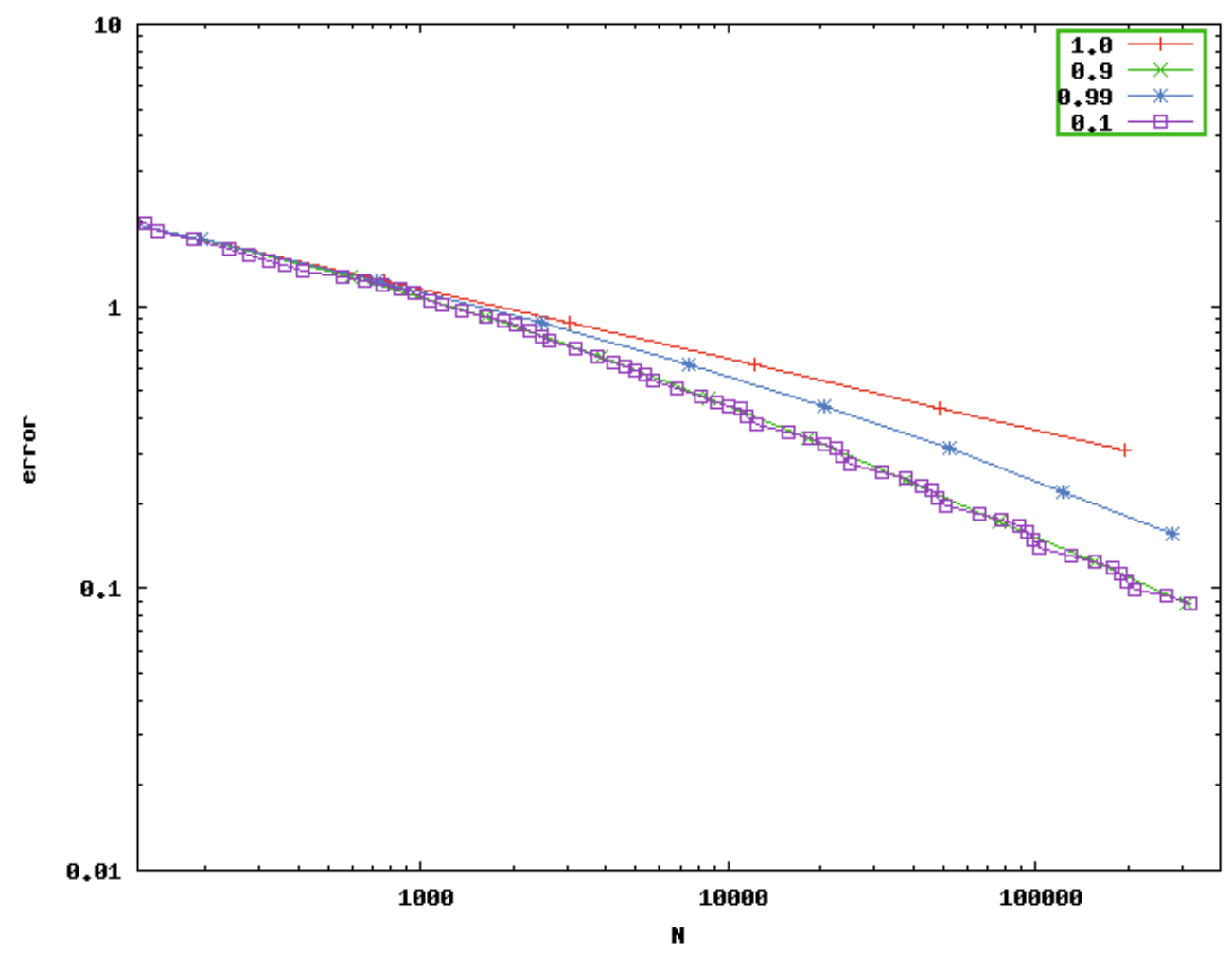

Figure 5. Decrease of the estimator for Example 2.

\section{REFERENCES}

[1] I. Babuška and W.C. Rheinboldt, Error estimates for adaptive finite element computations. SIAM J. Numer. Anal. 15 (1978) 736-754.

[2] R. Becker and S. Mao, An optimally convergent adaptive mixed finite element method. Numer. Math. 111 (2008) 35-54.

[3] R. Becker and D. Trujillo, Convergence of an adaptive finite element method on quadrilateral meshes. Research Report RR-6740, INRIA, France (2008).

[4] R. Becker, C. Johnson and R. Rannacher, Adaptive error control for multigrid finite element methods. Computing 55 (1995) $271-288$.

[5] R. Becker, S. Mao and Z.-C. Shi, A convergent adaptive finite element method with optimal complexity. Electron. Trans. Numer. Anal. 30 (2008) 291-304.

[6] P. Binev, W. Dahmen and R. DeVore, Adaptive finite element methods with convergence rates. Numer. Math. 97 (2004) 219-268.

[7] J.H. Bramble and J.E. Pasciak, New estimates for multilevel algorithms including the v-cycle. Math. Comp. 60 (1995) $447-471$.

[8] C. Carstensen, Quasi-interpolation and a posteriori error analysis in finite element methods. ESAIM: M2AN 33 (1999) $1187-1202$.

[9] C. Carstensen and R. Verfürth, Edge residuals dominate a posteriori error estimates for low order finite element methods. SIAM J. Numer. Anal. 36 (1999) 1571-1587.

[10] J.M. Cascon, Ch. Kreuzer, R.N. Nochetto and K.G. Siebert, Quasi-optimal convergence rate for an adaptive finite element method. SIAM J Numer. Anal. 46 (2008) 2524-2550.

[11] P.G. Ciarlet, The finite element method for elliptic problems, Studies in Mathematics and its Applications 4. Amsterdam, New York, Oxford: North-Holland Publishing Company (1978).

[12] A. Cohen, W. Dahmen and R. DeVore, Adaptive wavelet methods for elliptic operator equations: Convergence rates. Math. Comput. 70 (2001) 27-75.

[13] R. DeVore, Nonlinear approximation. Acta Numer. 7 (1998) 51-150. 
[14] W. Dörfler, A convergent adaptive algorithm for Poisson's equation. SIAM J. Numer. Anal. 33 (1996) 1106-1124.

[15] W. Dörfler and R.H. Nochetto, Small data oscillation implies the saturation assumption. Numer. Math. 91 (2002) 1-12.

[16] K. Eriksson, D. Estep, P. Hansbo and C. Johnson, Introduction to adaptive methods for differential equations. Acta Numer. 4 (1995) 105-158.

[17] P. Morin, R.H. Nochetto and K.G. Siebert, Data oscillation and convergence of adaptive FEM. SIAM J. Numer. Anal. 38 (2000) 466-488.

[18] P. Morin, K.G. Siebert and A. Veeser, A basic convergence result for conforming adaptive finite elements. Math. Models Methods Appl. Sci. 18 (2008) 707-737.

[19] R. Stevenson, Optimality of a standard adaptive finite element method. Found. Comput. Math. 7 (2007) 245-269.

[20] R. Verfürth, A review of a posteriori error estimation and adaptive mesh-refinement techniques. John Wiley/Teubner, New York-Stuttgart (1996).

[21] H. Wu and Z. Chen, Uniform convergence of multigrid v-cycle on adaptively refined finite element meshes for second order elliptic problems. Sci. China Ser. A 49 (2006) 1405-1429. 